\title{
Wedge Mode Propagation Characteristics of Triangular-shaped Surface Plasmon Waveguide
}

\author{
Nguyen Van Chinh, Nguyen Thanh Huong, Chu Manh Hoang* \\ International Training Institute for Materials Science, Hanoi University of Sciences and Technology, \\ No.1, Dai Co Viet, Hai Ba Trung, Hanoi, Vietnam
}

Received 15 September 2016

Revised 28 September 2016; Accepted 30 September 2016

\begin{abstract}
In this paper, we investigate wedge mode propagation characteristics of a triangularshaped surface plasmon waveguide. This structure consists of a thin metal layer deposited onto the surface of a triangular-shaped silicon waveguide which could be fabricated on a silicon-oninsulator wafer by wet-bulk micromachining. These elements are embedded in a dielectric medium (such as air) to form metal-dielectric interface and the surface plasmon wave propagates at the wedge of metal layer. The influence of structural parameters such as metal layer, height of silicon waveguide and effect of fabrication on the wedge mode propagation characteristics of the waveguide is analyzed by using numerical simulation.
\end{abstract}

Keywords: V-shaped waveguide, Surface plasmon polariton, plasmonic waveguide.

\section{Introduction}

Surface Plasmon Polariton (SPP) is surface electromagnetic wave which propagates at the metaldielectric interface. It is the result of the strong coupling between light and collective oscillation of free electron at a metal surface. In recent decades, the study on the theories and applications of SPP is growing fast and separated in a new field - Plasmonics. As a surface wave, the propagation of SPP is highly sensitive to the roughness and the electromagnetic field is confined in the space near the interface of the medium. There are many studies on novel devices to apply the sensitivity of SPP for biosensors [1-3]. In these structures, the interaction of biological reagents will change the surface properties of devices. Therefore, the propagation characteristics of SPP will be changed. One of other valuable properties of SPP, which makes it so interesting, is the confinement of wave in nano-scale. To achieve photonic circuits with high integration density, it is necessary to reduce the size of elements such as structures to guide optical signal. Three kinds of typical nanophotonic waveguides have been developed, which includes nanophotonic wires [4, 5], photonic-crystal waveguides [6] and nanoplasmonic waveguides [7]. The former two nanophotonic waveguides, which utilize nanostructures with ultra-high index contrast, are limited due to the classical optical diffraction phenomena. In contrast, a nanoplasmonic waveguide can break the diffraction limit and enable deep sub-

\footnotetext{
${ }^{*}$ Corresponding author. Tel.: 84-4-38680787

Email: hoangcm@itims.edu.vn
} 
wavelength confinement and wave-guiding of light, which makes it become a very attractive candidate for ultra-high integration density. Numerous of nanoplasmonic waveguides have been proposed and demonstrated in past years, including metal nano-slot waveguides [8], strip waveguides [9], metal Vgroove waveguides [10], wedge waveguides [5], and hybrid waveguides [11][12]. Among these, the wedge plasmon polariton (WPP) waveguides are the most prominent about the possibility of electromagnetic field confinement. In this paper, we will study about a WPP waveguide that can be fabricated by wet - bulk micromachining.

\section{Model of the surface plasmon polariton waveguide}

Structure of surface plasmon polariton waveguide is shown in Fig. 1. This structure consists of a triangular silicon waveguide, which is fabricated on a Silicon-on-Insulator (SOI) wafer. A noble metal layer is deposited onto the surface of the waveguide to form metal-dielectric interface. The silicon waveguide forms a mold to deposit a metal layer in an inverted V-like shape. The interface between the V-shaped metal layer and air medium is used for guiding surface plasmon wave. The surface plasmon polariton (WPP) mode propagates on the top wedge of structure. We will evaluate the dependence of propagation characteristics on the change of structural parameters such as deposited metal layer and dimensions of silicon waveguide.

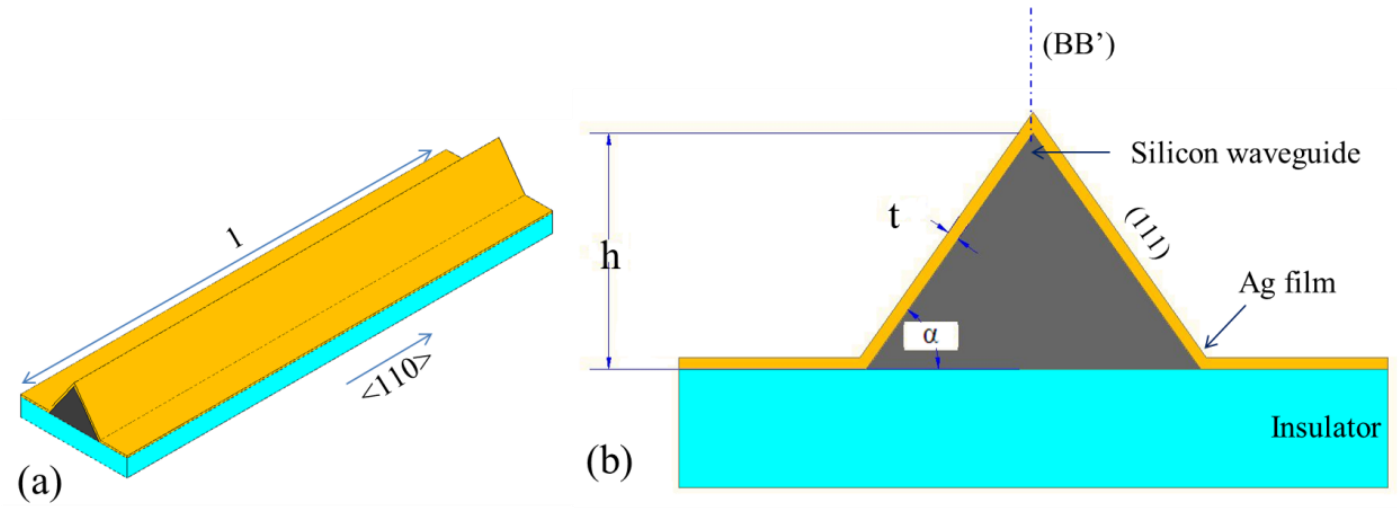

Fig 1. Schematic of triangular-shaped plasmonic waveguide used for simulation: (a) a 3D view of the waveguide and (b) cross-section of the waveguide.

The Maxwell theory describes the expression of SPPs wave on a metal-dielectric interface by [13]

$$
\mathbf{E}=\mathbf{E}_{0} \mathrm{e}^{\mathrm{i}\left(\mathrm{k}_{\mathrm{T}}^{\mathrm{x}}+\mathrm{k}_{\mathrm{n}} \mathrm{x}-\omega \mathrm{t}\right)}
$$

with the effective refractive index and skin depth

$$
\begin{aligned}
& \mathbf{n}_{\text {spp }}=\sqrt{\frac{\varepsilon_{\mathrm{m}}^{\prime} \varepsilon_{\mathrm{d}}}{\varepsilon_{\mathrm{m}}^{\prime}+\varepsilon_{\mathrm{d}}}} \\
& \delta_{2}=\mathbf{k}_{\mathrm{o}} \sqrt{\frac{\varepsilon_{1}^{\prime}+\varepsilon_{2}}{\varepsilon_{2}^{2}}}
\end{aligned}
$$

Using parameters in Table 1, we obtain the effective refractive index and mode size of SPPs propagating along the silver-air interface being 1.0038 and $2.86 \mu \mathrm{m}$, respectively. 
On a metal tip, WPP is the coupling mode formed by two SPP waves propagating toward the tip on the two opposite faces of the wedge [14]. These SPPs stop at the tip of wedge with both their phase and group velocities. The group velocity tends to zero and the wave vector tends to infinite at the tip. This leads to infinitesimal mode size (nanofocusing) and the wave can be propagated on very large distance. However, this model is applied only to very small wedge angle $\left(7^{\circ}\right)$. For the large wedge angle $\left(70.6^{\circ}\right)$, we must use numerical simulation methods to solve.

Table 1. Parameters used for simulating SPP mode

\begin{tabular}{lll}
\hline Symbol & Value & Difination \\
\hline$\lambda$ & $1.55 \mu \mathrm{m}$ & Wavelength in vacuum \\
1 & $10 \mu \mathrm{m}$ & Length of waveguide \\
$\mathrm{h}$ & $1 \mu \mathrm{m}$ & Height of waveguide \\
$\mathrm{n}_{\mathrm{Si}}$ & 3.4757 & Refractive index of silicon \\
$\mathrm{n}_{\mathrm{SiO} 2}$ & 1.4957 & Refractive index of silicon dioxide layer \\
$\mathrm{n}_{\mathrm{Ag}}$ & $0.15649-11.567 \mathrm{i}$ & Complex refractive index of Silver \\
$\mathrm{n}_{\mathrm{Au}}$ & $0.23823-11.263 \mathrm{i}$ & Complex refractive index of Gold \\
$\alpha$ & $54.7^{\circ}$ & Sidewall angle \\
$\mathrm{t}$ & $(10: 10: 200) \mathrm{nm}$ & Thickness of metal layer \\
\hline
\end{tabular}

By finite element method, we can find the propagation constant of WPP mode, that satisfies eigenvalue equations

$$
\begin{aligned}
& \left(\nabla-\mathrm{ik}_{1}\right) \times\left(\left(\nabla-\mathrm{ik}_{1}\right) \times \mathrm{E}_{1}\right)-\mathrm{k}_{\mathrm{o}}{ }^{2} \varepsilon_{\mathrm{r}} \mathrm{E}_{1}=0 \\
& \left(\nabla-\mathrm{ik}_{2}\right) \times\left(\left(\nabla-\mathrm{ik}_{2}\right) \times \mathrm{E}_{2}\right)-\mathrm{k}_{\mathrm{o}}{ }^{2} \varepsilon_{\mathrm{r}} \mathrm{E}_{2}=0
\end{aligned}
$$

In our study, we used software COMSOL, a commercial product for academic purpose to solve these equations. The model is three - dimension with the calculation size is $4 \times 4 \times 10 \mu \mathrm{m}^{3}$. Two boundary ports are applied on the back and front face of the waveguide to analyze modes that can propagate in structure. The perfectly matched layer is larger than five times the calculation domain and the minimum mesh size is about 1 nanometer (at near the wedge of waveguide).

\section{Results and discussion}

Firstly, we investigate the influence of metal layer on the propagation of plasmon wave on the waveguide. In this study, we use two metals being gold and silver, which are common in plasmonic technology, to simulate. The metal layer can be deposited onto triangular silicon waveguide by sputtering technique. The thickness is studied in each step of $10 \mathrm{~nm}$ in the range from $10 \mathrm{~nm}$ to 200 $\mathrm{nm}$. To ensure that the thickness of metal film is the same throughout the structure, the wedge of metal is filleted with a radius equal to the thickness of the metal layer. 

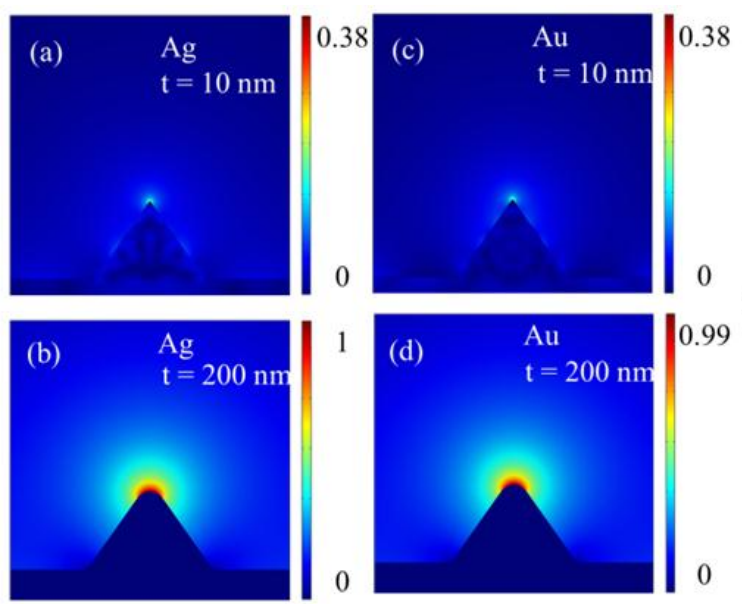

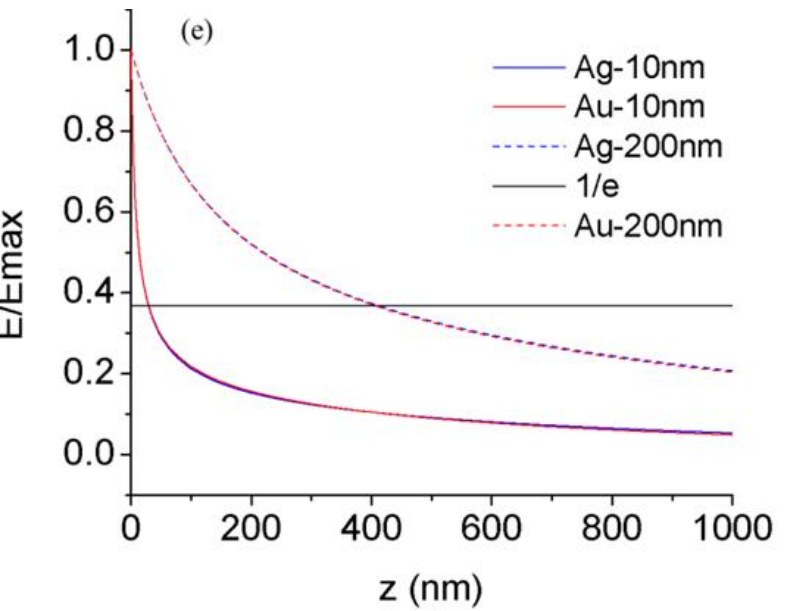

Fig 2. Distribution of normalized electric field in the cross-section of waveguide at various thicknesses of silver and gold layer: (a)-(b) for Ag with the thicknesses of $10 \mathrm{~nm}$ and 200nm respectively and (c)-(d) for Au with the thicknesses of $10 \mathrm{~nm}$ and 200nm respectively; (e) Distribution of normalized Electric field in air along the (BB') cutline as shown in Fig. 1 (b).

Figure 2 (a)-(d) shows the distributions of electric field that are normalized with respect to maximum field value. For both metals, the maximum electric field values are almost the same. When the thickness of metal layer increases, the maximum electric field values also increase. The electric field distributes in air medium, and concentrates mainly on the area round the wedge of the waveguide. This electric field reaches the maximum value at metal surface and declines rapidly when it is away from the surface. Figure 2 (e) describes more clearly about the sharp decline of the field along (BB') cutline (shown in Fig. 1). The field falls off exponentially in air, the intersection of these curves with 1/e line giving a quantitative estimation of the confinement of electromagnetic energy. As seen in Fig. 2 (e), the mode size is almost constant for two metals. In the case of very thin metal layer $(10 \mathrm{~nm})$, the field falls off to 1/e at a distance of only a few tens of nanometers. Otherwise, in the case of thick metal film (200nm), the mode size increases to about $500 \mathrm{~nm}$. Thus, the confinement of waveguide mode does not depend on the nature of metal, but depends strongly on the thickness of metal layer.

The propagation characteristics of the waveguide at various metal thicknesses are shown in Fig. 3. Here, we evaluate three feature parameters being effective mode index $n_{\text {eff }}$ (the ratio of wave number of WPP mode and wave number in vacuum), propagation length (the distance at which the field intensity decreases e time) and mode area (area bounded by a curve where electric field falls off to 1/e) (Figs. 3 (a)-(c)). Figure 3 (d) shows the shape of mode area (the red area at the wedge of waveguide). When the metal thickness increases, the effective mode index decreases and the propagation length increases. Both these quantities tend asymptotically to a value corresponding to the infinite thickness. The mode area of gold and silver are almost the same and increase strongly with the thickness. With the same structural parameters, the attenuation using gold is stronger than using silver about two times, while other characteristics are almost the same. So, we choose silver for forming the metaldielectric interface for the following study.

As seen in Fig. 3 (b), at the thickness $t=20 \mathrm{~nm}$, the propagation length has a minimum value. It might be due to the coupling of WPP with a high order mode in silicon waveguide. Increasing the thickness of metal layer, the coupling decreases and will be negligible at thickness larger than $150 \mathrm{~nm}$. 

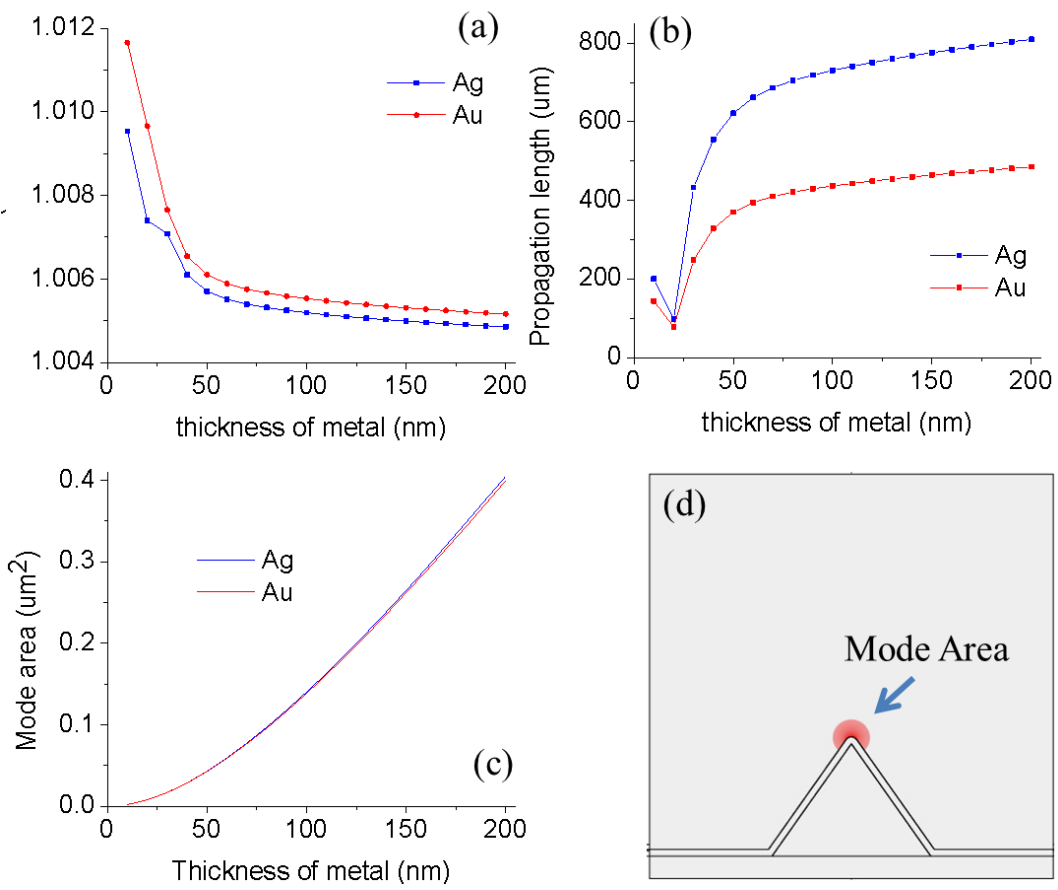

Fig 3. Propagation characteristics of WPP mode at various thicknesses of metal (a)-(c) and the shape of mode area $(d)$.
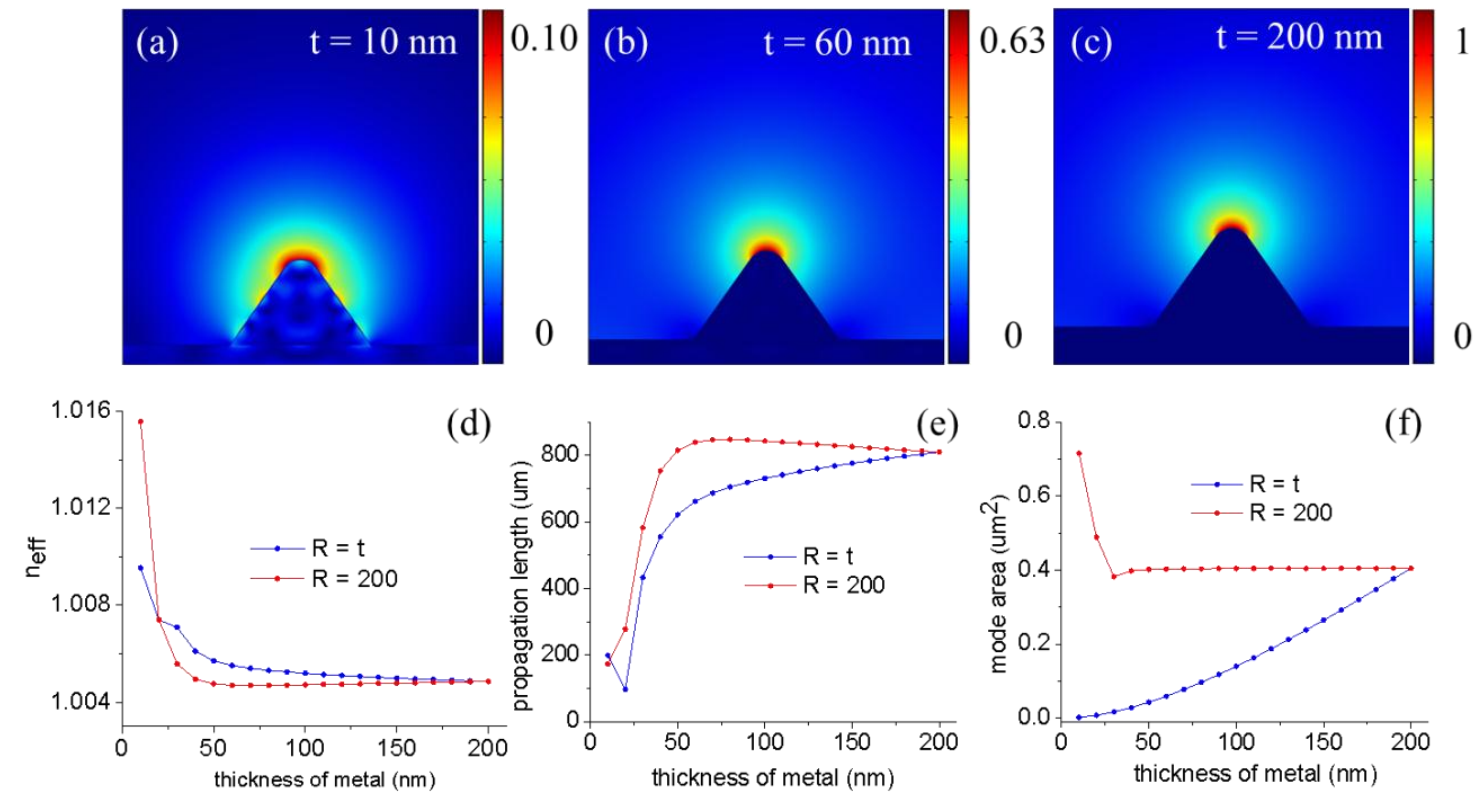

Fig 4. (a)-(c) are distributions of electric field, when we fillet the top corner of silicon waveguide; (d)-(f) are propagation chracteristics of waveguide at various thicknesses of metal, in which $\mathrm{R}=\mathrm{t}$ (blue line) and $\mathrm{R}=200$ $\mathrm{nm}$ (red line) are for the plasmonic waveguide with and without the silicon waveguide filleted at the top wedge corner, respectively. 
In the above study, we have filleted the wedge of metal layer with radius equal the value of metal thickness. It leads to the changing of wedge mode area that concentrates the electromagnetic energy, thus the propagation of WPP mode is changed. To keep the wedge radius of metal constant, we have to fillet the top wedge corner of silicon waveguide with the appropriate value of radius. Figure 4 shows the simulation results with the radius of metal wedge is always $200 \mathrm{~nm}$. For the homogenization of metal layer on the entire structure, we set the filleted radius of silicon waveguide is $(200-t) \mathrm{nm}$ with respective to the thickness of metal layer t. Figures 4 (a)-(c) displays the distribution of electromagnetic field with three metal thicknesses, $\mathrm{t}=10 \mathrm{~nm}, 60 \mathrm{~nm}$, and $200 \mathrm{~nm}$, respectively. If we increase the thickness of metal, the maximum value of field will be increased, which is the same as the wedge of silicon waveguide not filled. However, the propagation properties in this case are slightly different (Figs.4 (d)-(f)). For comparison, the propagation mode characteristics of the silicon waveguide without filleting the top wedge corner are also shown in Figs. 4 (d)-(f). The propagation length is no longer a minimum value at $20 \mathrm{~nm}$ and reaches a maximum value at

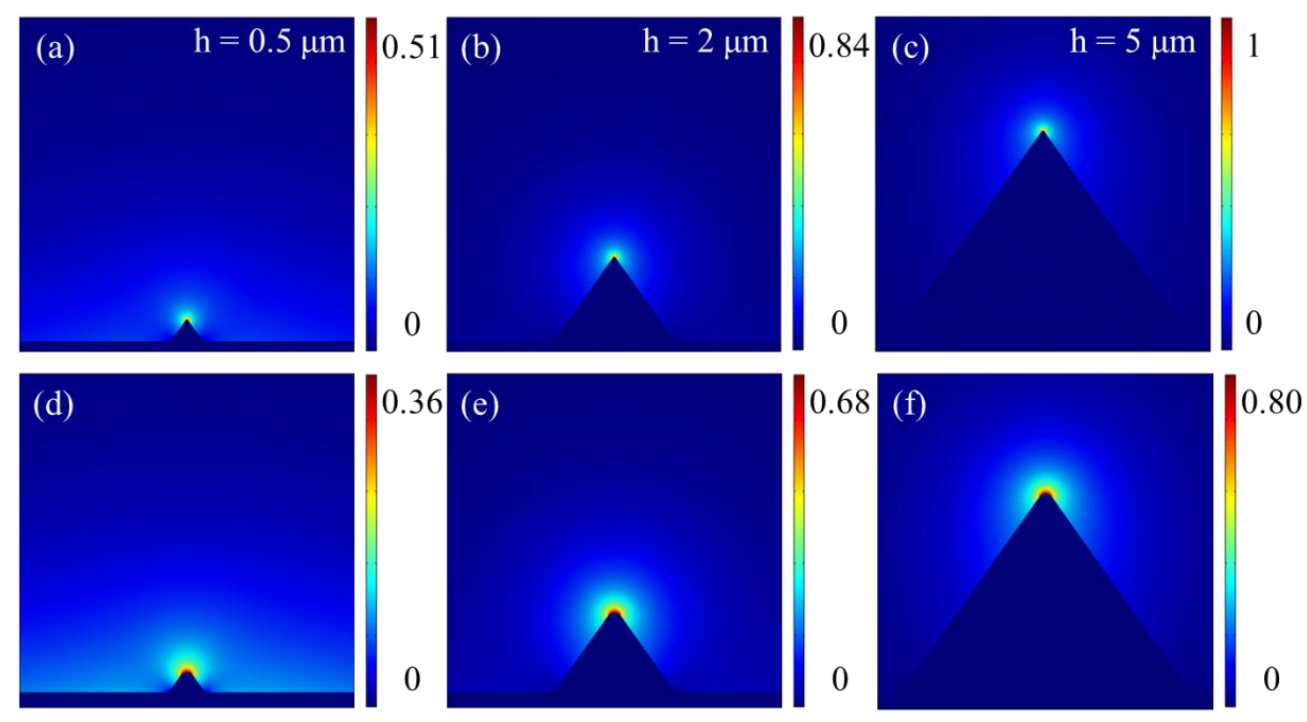

Fig 5. Electromagnetic field of WPP mode distributes on the wedge of waveguide at different heights in two cases $\mathrm{t}=60 \mathrm{~nm}(\mathrm{a})-(\mathrm{c})$ and $200 \mathrm{~nm}(\mathrm{~d})-(\mathrm{f})$.

the thickness of $60 \mathrm{~nm}$. With the same thickness, the attenuation in structure with the filleted top wedge corner of silicon waveguide is smaller than in the remaining structure. It may be due to the decreasing of confinement of WPP mode. This investigation on the filleting effect is a guideline for predicting the operation characteristics of the waveguide when there is variation in fabrication condition.

The dependence of WPP mode on the height $\mathrm{h}$ of triangular cross-section of the waveguide is studied in next section. In the fabrication using wet-bulk micromachining on SOI wafer, the height of waveguide approximates the thickness of device layer. So, by choosing SOI wafers having different device layer thicknesses, we can change the height of the waveguide. The parameters used for simulation are taken from Table 1 while $\mathrm{h}$ is varied from $0.5 \mu \mathrm{m}$ to $5 \mu \mathrm{m}$. Figure 5 displays the distribution of electromagnetic field at various values of $\mathrm{h}$ in which the thicknesses of metal layer are $60 \mathrm{~nm}$ for Figs. (a)-(c) and 200nm for Figs. (d)-(f). In waveguides with large height $(\mathrm{h}=5 \mu \mathrm{m}$ ), electromagnetic field concentrates mainly on the wedge of the waveguide. In waveguides with low height ( $h \leq 0.5 \mu \mathrm{m}$ ), the field not only distributes on the wedge but also spreads over the surface of substrate. 

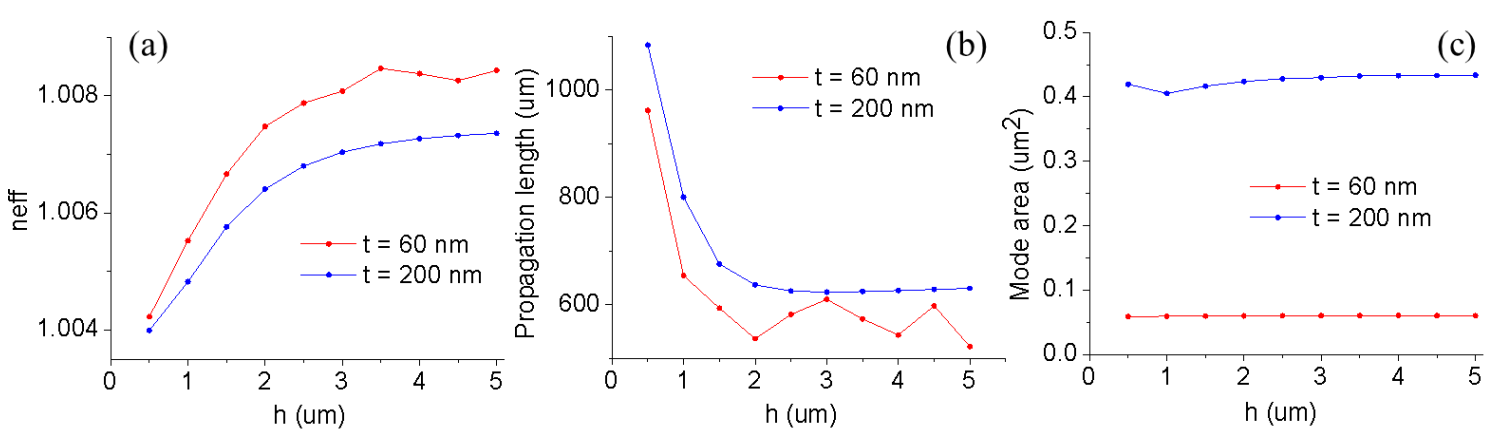

Fig 6. The propagation characteristics of waveguide are investigated as a function of $h$ when $\mathrm{t}=60 \mathrm{~nm}$ (red line) and 200nm (blue line).

The propagation characteristics depending on the height of the waveguide are shown in Fig. 6. In the case of large metal thickness, when the height increases, the wavenumber $\left(\mathrm{n}_{\mathrm{eff}}\right)$ and the attenuation $\left(1 / \mathrm{L}_{\mathrm{wpp}}\right)$ increase and they approach to special values when $\mathrm{h}$ is more than $2 \mu \mathrm{m}$. In the case that the thickness is $60 \mathrm{~nm}$, the coupling with high order mode in the silicon waveguide makes the curve fluctuate, but the shape is the same. Approximately, we can explain that phenomena as follow: the WPP mode is the result of coupling between two SPPs waves that propagate on two sidewalls of the waveguide. This coupling is like the coupling in a thin metal film with the variation of thickness. It makes wavenumber increase, but exchanged by the increase of loss. When the height is more than $2 \mu \mathrm{m}$, the film becomes so thick that the coupling is negligible and only the part nears the wedge contributing to the coupling. This causes the saturation of wavenumber and propagation length of WPP mode in the waveguide. When $\mathrm{h}$ is too small, the coupling becomes weak and the effective mode index of WPP mode approximates the mode index of SPPs $\left(\mathrm{n}_{\mathrm{spp}}=1.0038\right)$.

\section{Conclusion}

We have investigated in detail propagation characteristics of the wedge surface plasmon waveguide depending on geometrical parameters and deposited metal. The investigated results show that the mode confinement and propagation length strongly depend on the thickness of metal layer. The mode confinement decreases when the thickness of metal layer increases. When the height of the waveguide increases, the mode size and propagation length decrease.

\section{Acknowledgments}

This research is funded by Vietnam National Foundation for Science and Technology Development (NAFOSTED) under grant number "103.02-2015.86".

\section{References}

[1] Brolo, A.: Plasmonics for future biosensors. Nat. Photonics. 6, 709-713 (2012).

[2] Gao, Y., Gan, Q., Xin, Z., Cheng, X., Bartoli, F.J.: Plasmonic Mach-Zehnder interferometer for ultrasensitive onchip biosensing. ACS Nano. 5, 9836-9844 (2011). 
[3] Rodrigo, D., Limaj, O., Janner, D., Etezadi, D., Abajo, F.J.G. De, Pruneri, V., Altug, H.: Mid-infrared plasmonic biosensing with graphene. Science, 349, 165-168 (2015).

[4] Han, Z., Bozhevolnyi, S.I.: Radiation guiding with surface plasmon polaritons. Rep. Prog. Phys. 76, 16402 (2013).

[5] Moreno, E., Rodrigo, S.G., Bozhevolnyi, S.I., Martín-Moreno, L., García-Vidal, F.J.: Guiding and focusing of electromagnetic fields with wedge plasmon polaritons. Phys. Rev. Lett. 100, 1-4 (2008).

[6] Pile, D.F.P., Ogawa, T., Gramotnev, D.K., Okamoto, T., Haraguchi, M., Fukui, M., Matsuo, S.: Theoretical and experimental investigation of strongly localized plasmons on triangular metal wedges for subwavelength waveguiding. Appl. Phys. Lett. 87, 1-4 (2005).

[7] Bian, Y., Gong, Q.: Deep-subwavelength light confinement and transport in hybrid dielectric-loaded metal wedges. Laser Photonics Rev. 8, 549-561 (2014).

[8] Dionne, J.A., Sweatlock, L.A., Atwater, H.A., Polman, A.: Plasmon slot waveguides: Towards chip-scale propagation with subwavelength-scale localization. Phys. Rev. B - Condens. Matter Mater. Phys. 73, 1-9 (2006).

[9] Gao, L., Tang, L., Hu, F., Guo, R., Wang, X., Zhou, Z.: Active metal strip hybrid plasmonic waveguide with low critical material gain. Opt. Express. 20, 11487 (2012).

[10] Dintinger, J., Martin, O.J.F.: Channel and wedge plasmon modes of metallic V-grooves with finite metal thickness. Opt. Express. 17, 2364-2374 (2009).

[11] Bian, Y., Gong, Q.: Low-loss hybrid plasmonic modes guided by metal-coated dielectric wedges for subwavelength light confinement. Appl. Opt. 52, 5733-41 (2013).

[12] Gui, C., Wang, J.: Wedge hybrid plasmonic THz waveguide with long propagation length and ultra-small deepsubwavelength mode area. Sci. Rep. 5, 11457 (2015).

[13] Raether, H.: Surface plasmons on smooth and rough surfaces and on gratings, Springer Tracts in Modern Physics, Vol. 111. (1988).

[14] Gramotnev, D.K., Vernon, K.C.: Adiabatic nano-focusing of plasmons by sharp metallic wedges. Appl. Phys. B Lasers Opt. 86, 7-17 (2007). 